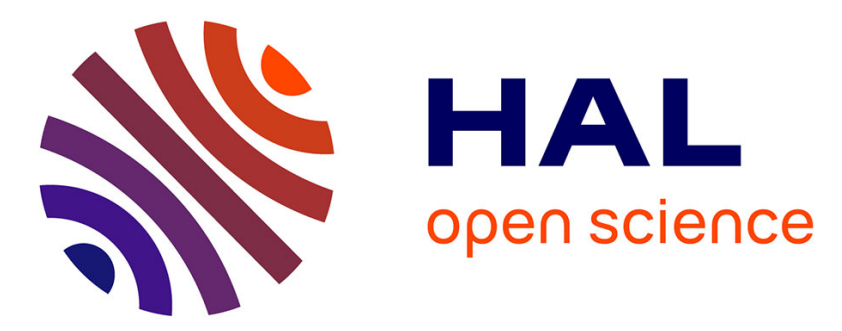

\title{
Development of a wavefront sensor for terahertz pulses
} Emmanuel Abraham, Harsono Cahyadi, Mathilde Brossard, Jérôme Degert, Eric Freysz, Takeshi Yasui

\section{To cite this version:}

Emmanuel Abraham, Harsono Cahyadi, Mathilde Brossard, Jérôme Degert, Eric Freysz, et al.. Development of a wavefront sensor for terahertz pulses. Optics Express, 2016, 24 (5), pp.5203-5211. 10.1364/OE.24.005203.v002 . hal-01297343

\section{HAL Id: hal-01297343 \\ https://hal.science/hal-01297343}

Submitted on 4 Apr 2016

HAL is a multi-disciplinary open access archive for the deposit and dissemination of scientific research documents, whether they are published or not. The documents may come from teaching and research institutions in France or abroad, or from public or private research centers.
L'archive ouverte pluridisciplinaire HAL, est destinée au dépôt et à la diffusion de documents scientifiques de niveau recherche, publiés ou non, émanant des établissements d'enseignement et de recherche français ou étrangers, des laboratoires publics ou privés.

\section{(1) $\$(0)$}

Distributed under a Creative Commons Attribution - NonCommercial - ShareAlikel 4.0 


\title{
Development of a wavefront sensor for terahertz pulses
}

\author{
Emmanuel Abraham, ${ }^{1,2, *}$ Harsono Cahyadi, ${ }^{4}$ Mathilde Brossard, ${ }^{1,2,3}$ Jérôme Degert, ${ }^{1,2}$ \\ Eric Freysz $^{1,2}$ and Takeshi Yasui ${ }^{4}$ \\ ${ }^{1}$ Univ. Bordeaux, LOMA, UMR 5798, F-33400 Talence, France \\ ${ }^{2}$ CNRS, LOMA, UMR 5798, F-33400 Talence, France \\ ${ }^{3}$ NeTHIS - New Terahertz imaging Systems, Mérignac, France \\ ${ }^{4}$ Institute of Technology and Science, Tokushima University, Tokushima 770-8506, Japan \\ "emmanuel.abraham@u-bordeaux.fr
}

\begin{abstract}
Wavefront characterization of terahertz pulses is essential to optimize far-field intensity distribution of time-domain (imaging) spectrometers or increase the peak power of intense terahertz sources. In this paper, we report on the wavefront measurement of terahertz pulses using a Hartmann sensor associated with a 2D electro-optic imaging system composed of a ZnTe crystal and a CMOS camera. We quantitatively determined the deformations of planar and converging spherical wavefronts using the modal Zernike reconstruction least-squares method. Associated with deformable mirrors, the sensor will also open the route to terahertz adaptive optics.
\end{abstract}

OCIS codes: (120.0280) Remote sensing and sensors; (110.6795) Terahertz imaging; (070.7345) Wave propagation.

\section{References and links}

1. J. Hartmann, "Bemerkungen über den Bau und die Justierung von Spektrographen," Z. Instrumentenkd. 20, 4758 (1900).

2. R. V. Shack and B. C. Platt, "Production and use of a lenticular Hartmann screen," J. Opt. Soc. Am. 61, 656-660 (1971).

3. B. C. Platt and R. Shack, "History and principles of Shack-Hartmann wavefront sensing," J. Refract. Surg. 17(5), S573-S577 (2001).

4. A. Bitzer, H. Helm, and M. Walther, "Beam-profiling and wavefront-sensing of THz pulses at the focus of a substrate-lens," IEEE J. Sel. Top. Quantum Electron. 14, 476-481 (2008).

5. M. Cui, J. N. Hovenier, Y. Ren, A. Polo, and J. R. Gao, "Terahertz wavefronts measured using the Hartmann sensor principle," Opt. Express 20(13), 14380-14391 (2012).

6. M. Cui, J. N. Hovenier, Y. Ren, N. Vercruyssen, J. R. Gao, T. Y. Kao, Q. Hu, and J. L. Reno, "Beam and phase distributions of a terahertz quantum cascade wire laser," Appl. Phys. Lett. 102(11), 111113 (2013).

7. H. Richter, M. Greiner-Bär, N. Dessmann, J. Pfund, M. Wienold, L. Schrottke, R. Hey, H. T. Grahn, and H.-W. Hübers, "Terahertz wavefront measurement with a Hartmann sensor," Appl. Phys. Lett. 101(3), 031103 (2012).

8. J. F. Molloy, M. Naftaly, and R. A. Dudley, "Characterization of Terahertz beam profile and propagation," IEEE J. Sel. Top. Quantum Electron. 19, 8401508 (2013).

9. Q. Wu and X. C. Zhang, "Free space electro-optic sampling of terahertz beams," Appl. Phys. Lett. 67(24), 3523 (1995).

10. N. V. Petrov, A. A. Gorodetsky, and V. G. Bespalov, "Holography and phase retrieval in terahertz imaging," Proc. SPIE 8846, 88460S (2013).

11. J. W. Goodman, Introduction to Fourier Optics (McGraw-Hill, 1968).

12. W. H. Southwell, "Wave-front estimation from wave-front slope measurements," J. Opt. Soc. Am. 70(8), 998$1006(1980)$.

13. T. Yasui, K. Sawanaka, A. Ihara, E. Abraham, M. Hashimoto, and T. Araki, "Real-time terahertz color scanner for moving objects," Opt. Express 16(2), 1208-1221 (2008).

14. M. Jewariya, E. Abraham, T. Kitaguchi, Y. Ohgi, M. A. Minami, T. Araki, and T. Yasui, "Fast three-dimensional terahertz computed tomography using real-time line projection of intense terahertz pulse," Opt. Express 21(2), 2423-2433 (2013)

15. P. Klarskov, A. C. Strikwerda, K. Iwaszczuk, and P. U. Jepsen, "Experimental three-dimensional beam profiling and modeling of a terahertz generated from a two-color air plasma," New J. Phys. 15(7), 075012 (2013). 


\section{Introduction}

The Hartmann sensor was invented more than a century ago for optical beam analysis [1]. It makes it possible to locally measure the wavefront slopes of an incoming optical radiation by using a mask composed of a circular holes array placed just in front of a 2D sensor. It was improved in 1970 by Shack who replaced the mask by a lens array [2]. At present, in the visible spectral region, commercially available Shack-Hartmann sensors can perform wavefront characterization for adaptive optics and a wide variety of applications including astronomy, ophthalmology and microscopy. These sensors are widely used to measure optical aberrations such as astigmatism, coma, spherical aberration, etc [3]. In the terahertz (THz) spectral domain, it is still challenging to fully measure the spatial profile and wavefront of a $\mathrm{THz}$ beam due to the lack of effective $\mathrm{THz}$ cameras.

A few previous studies were oriented towards the measurement of $\mathrm{THz}$ wavefronts for both continuous wave and pulsed sources. In 2008, Bitzer et al. determined the beam profiles of THz pulses after passing through a hyperhemispherical silicon lens [4]. They observed an asymmetric spatial-temporal field dynamic in the focus of the lens attributed to a distortion of the incident $\mathrm{THz}$ wavefront. However, they did not measure the $\mathrm{THz}$ wavefront but only the $\mathrm{THz}$ beam profile and their indirect and time-consuming method used a xy-scanning system in order to determine point-by-point the beam profile. In 2012, Cui et al. proposed the first $\mathrm{THz}$ wavefront measurement associated with a Hartmann sensor and indirect xy-scanning method to detect the radiation with a pyroelectric sensor [5,6]. The same year, Richter and associates presented a similar arrangement with a $2 \mathrm{D}$ detection composed by a microbolometer camera [7]. However, this method cannot be applied with short THz pulses produced by femtosecond laser pulses owing to the low sensitivity of the thermal incoherent detector. Another aperture scanning device was proposed in 2013 to characterize the $\mathrm{THz}$ beam propagation in a time-domain spectrometer [8]. Associated with a Hartmann mask, it has been used to produce 2D topographical image of the THz wavefront. From these few previous works, we can conclude that only two studies have been proposed for the wavefront reconstruction of $\mathrm{THz}$ pulses produced by femtosecond laser pulses. Both approaches were mainly limited by a $2 \mathrm{D}$ aperture scanning $[4,8]$.

For $\mathrm{THz}$ pulse detection, it is well-known that spatial-temporal profiles of $\mathrm{THz}$ transient fields can be directly determined through electro-optic (EO) sampling in a nonlinear crystal [9]. By varying the time delay between the probe and THz pulses, EO sampling can record the spatial dependence of the full (amplitude and phase) THz electric field, $\mathrm{E}_{\mathrm{THz}}$, in the crystal plane. Then, from the phase variation of $\mathrm{E}_{\mathrm{THz}}$ in this plane, it is numerically possible to determine the surface of equi-phase, which constitutes the definition of the wavefront. This approach is close to the principle of $\mathrm{THz}$ pulse time-domain holography and the concept of phase retrieval in $\mathrm{THz}$ imaging [10]. However, the method requires to propagate the $\mathrm{THz}$ waves, using the Rayleigh-Sommerfeld equation or the angular spectrum algorithm [11], in order to calculate the location of the $\mathrm{THz}$ wavefront at the crystal position.

In this paper, derived from visible or infrared technology, we use a more standard method for $\mathrm{THz}$ wavefront measurement, which is based on the classical utilization of a Hartmann mask. This approach can appear less straightforward since it does not use the phase but the amplitude of $\mathrm{E}_{\mathrm{THz}}$ to measure the wavefront. However, it employs robust algorithms such as the modal reconstruction method to determine the final wavefront decomposed on the basis of the Zernike polynomials [12]. This calculation does not need any assumptions on the $\mathrm{THz}$ wave propagation. This constitutes the main advantage of the Hartmann method. The originality of our approach, compared to refs. [4] and [8], concerns the use of 2D measurement system which does not need to scan any aperture through the $\mathrm{THz}$ beam cross section. For that purpose, we don't use a $\mathrm{THz}$ camera but an indirect detection of the $\mathrm{THz}$ beam thanks to a $2 \mathrm{D} \mathrm{EO}$ imaging system [13]. It means that the $\mathrm{THz}$ wavefront to analyze is transferred to a visible laser beam that can be more simply analyzed using a visible camera. 
Combined with a Hartmann mask, this method has been applied to fully determine the frequency-resolved wavefront of THz pulses. Especially, the Zernike modal reconstruction method has been used to measure the wavefronts and optical aberrations of planar and convergent $\mathrm{THz}$ beams.

\section{Experimental setup}

The proposed method requires $\mathrm{THz}$ pulses with electric field strengths roughly larger than 1 $\mathrm{kV} / \mathrm{cm}$ for effective 2D EO sampling. Therefore, it requires an amplified femtosecond laser source and applies well with $\mathrm{THz}$ generation by laser-induced air plasma, optical rectification in nonlinear crystals $\left(\mathrm{ZnTe}, \mathrm{LiNbO}_{3}, \mathrm{DAST}\right)$ and large interdigitized photoconductive antennas. In our study, $\mathrm{THz}$ pulses were generated by optical rectification of amplified femtosecond laser pulses $(800 \mathrm{~nm}, 1 \mathrm{~mJ}, 150 \mathrm{fs})$ in a $1 \mathrm{~mm}$-thick ZnTe crystal with a clear aperture of $25 \mathrm{~mm}$ (Fig. 1(a)). The collimated THz beam is sent into the ZnTe crystal for collinear EO detection with a time-delayed laser probe pulse, reflected by the front face of the crystal. In the crystal, the spatial distribution of the broadband $(0.1-2 \mathrm{THz}) \mathrm{THz}$ electric field modifies the intensity distribution of the probe beam that is finally captured by a $232 \times 232$ pixels CMOS camera after passing through an analyzer and a lens that images the crystal plane (magnification $\gamma=-0.23$ ). Similar optical arrangement was previously used for realtime $2 \mathrm{D}$ and $3 \mathrm{D} \mathrm{THz}$ imaging [14]. For a given time delay between the $\mathrm{THz}$ and the probe pulses, the system is able to provide a $2 \mathrm{D}$ (x-horizontal and y-vertical directions) image at $800 \mathrm{~nm}$ corresponding to the distribution of the THz electric field at the crystal position. This image can be obtained with a $500 \mathrm{~Hz}$ acquisition rate thanks to the dynamic subtraction. However, signal-to-noise ratio higher than 100 usually requires an averaging time of $0.5 \mathrm{~s}$. By changing the time delay between the $\mathrm{THz}$ and probe pulses, it is possible to record the temporal evolution of this $\mathrm{THz}$ electric field. Thanks to this indirect $\mathrm{THz}$ imaging, our system will be able to analyze the spatial distribution of the $\mathrm{THz}$ electric field at the crystal position.

The Hartmann mask consists of a 1 mm-thick metallic plate with 9 by 9 circular holes ( 1 $\mathrm{mm}$ diameter with $2 \mathrm{~mm}$ periodicity in the horizontal and vertical directions). The mask is placed $10 \mathrm{~mm}$ in front of the ZnTe crystal, as indicated by the dashed line in Fig. 1(a). This distance $\mathrm{D}$ corresponds to a good compromise between $\mathrm{THz}$ beam diffraction and the wavefront slope measurement, as already noticed in refs. [5] and [7]. To confirm this mask design, we calculated the intensity distribution of the $\mathrm{THz}$ electric field at the distance $\mathrm{D}=10$ $\mathrm{mm}$ behind the mask, assuming a Fresnel-Kirchhoff wave propagation of an incident monochromatic plane wave at $1 \mathrm{THz}$. Figure 1(b) shows the corresponding simulated $\mathrm{THz}$ intensity distribution, confirming that the mask design is appropriated since each hole produces a clearly projected imaging spot in the crystal plane.

Figure 1(c) illustrates the deviations of the hole positions in the plane of the ZnTe crystal, induced by a $\mathrm{THz}$ wavefront distortion. It also shows the strategic role of the crystal as an intermediate plane between the mask and the CMOS camera. The displacement $\Delta \mathrm{x}$ of one hole centroid in the plane of the crystal is clearly converted into a displacement $\delta x=|\gamma| \Delta x$ at the position of the CMOS sensor. Similar deviations $\Delta y$ and $\delta y$ can be measured along the perpendicular y-direction. 


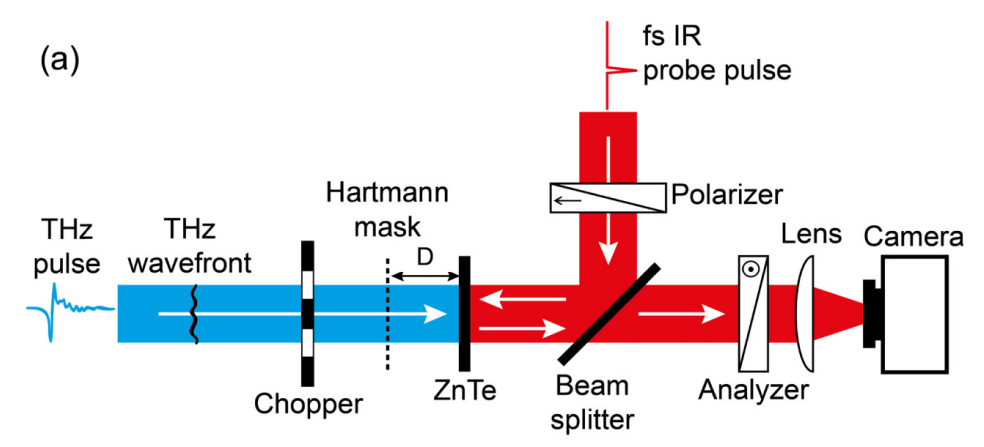

(b)
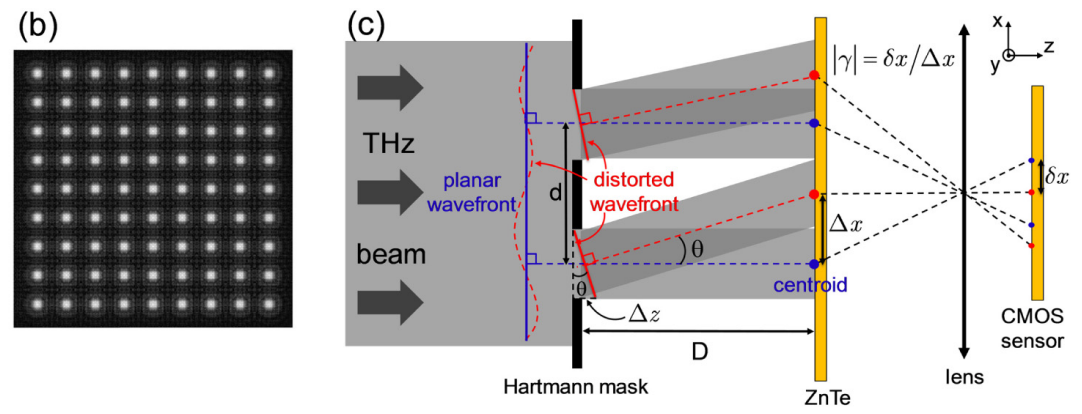

Fig. 1. (a) Experimental setup of the $\mathrm{THz}$ wavefront sensor. (b) Monochromatic $\mathrm{THz}$ plane wave $(1 \mathrm{THz})$ passing through the Hartmann mask (1 $\mathrm{mm}$ diameter with $2 \mathrm{~mm}$ periodicity). Simulation of the intensity distribution at the crystal position ( $\mathrm{D}=10 \mathrm{~mm}$ behind the mask). (c) Schematic representation of $\mathrm{THz}$ wavefront distortion and spot displacement. ZnTe crystal plane is imaged onto the CMOS sensor with magnification factor $\gamma=-0.23$. D $=10 \mathrm{~mm}, \mathrm{~d}=2$ $\mathrm{mm}$.

\section{Experimental results}

Before inserting the Hartmann mask, the THz pulse is directly sent onto the ZnTe crystal for EO sampling with the femtosecond laser probe pulse. The THz beam incident on the crystal is characterized by an expected planar wavefront. Then, we inserted a plan-convex THz lens (f' $=100 \mathrm{~mm}$ focal length) at $\mathrm{e}=65 \mathrm{~mm}$ before the crystal (not represented in Fig. 1). This second configuration provides a converging spherical $\mathrm{THz}$ beam at the crystal position with a theoretical $f^{\prime}-e=35 \mathrm{~mm}$ radius of curvature.

Figure 2(a) (resp. 2(c)) shows the transversal xy-image of the collimated planar (resp. converging spherical) $\mathrm{THz}$ beam for a $0 \mathrm{ps}$ time delay between the $\mathrm{THz}$ and probe pulses (corresponding to the main negative peak of the $\mathrm{THz}$ signal in the center of the image). Videos are available as supplementary materials to see the complete evolution of the transversal THz beam profile for different time delays. For both configurations, Fig. 2(b) and 2(d) show the temporal evolution of the $\mathrm{THz}$ pulse in the vertical $\mathrm{y}$-direction. These data make it possible to qualitatively assume that the $\mathrm{THz}$ wavefront is planar (resp. spherical and convergent), even if no quantitative optical aberrations can be measured at this stage. However, the radius of curvature of the spherical wavefront can be estimated as follows. From the temporal waveform of the THz electric field, as presented in Fig. 3, we can measure a time delay $\Delta \mathrm{t}=0.51 \mathrm{ps}$ between the main negative (blue color in Fig. 2(c)) and positive peaks (red color in Fig. 2(c)) of the THz pulse. Then, we measure the distance $r=3.13 \mathrm{~mm}$ between the center of the main blue circle and the radius of the circular red ring in Fig. 2(c), which corresponds to the intersection of the crystal plane with two spherical wavefronts, one for the negative peak and one for the positive peak (dashed line in Fig. 2(d)). Assuming a perfect spherical wavefront, the radius of curvature is given by: 


$$
R=\frac{r^{2}}{2 c \Delta t}
$$

where $\mathrm{c}$ is the speed of light. Our measurement gives $\mathrm{R}=32 \mathrm{~mm}$ which is close to the $35 \mathrm{~mm}$ theoretical value. The difference can be explained by optical aberrations that have not been taken into account in this first simple measurement.
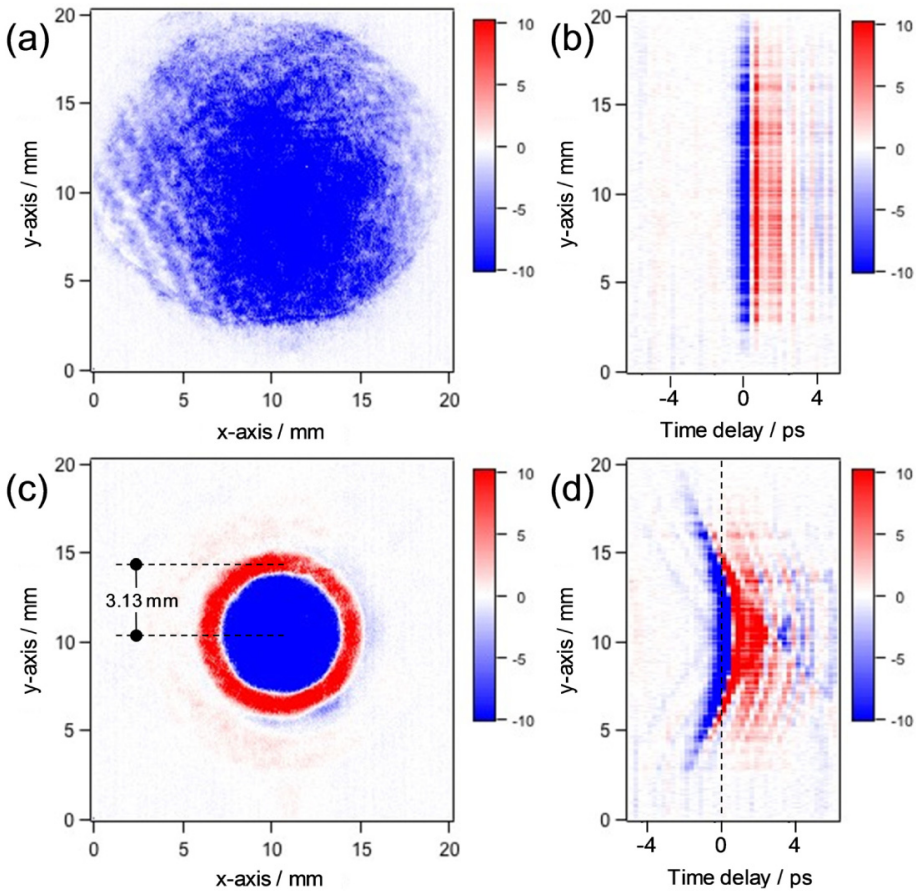

Fig. 2. (a) xy-image of a collimated planar THz beam for a 0 ps time delay. (b) Corresponding yt-image. (c) xy-image of a converging spherical $\mathrm{THz}$ beam for a $0 \mathrm{ps}$ time delay. (d) Corresponding yt- image. Red color is for a positive electric field and blue color is for a negative one. Associated supplementary materials: Visualization 1 corresponding to the temporal evolution of (a) and Visualization 2 to the temporal evolution of (c).

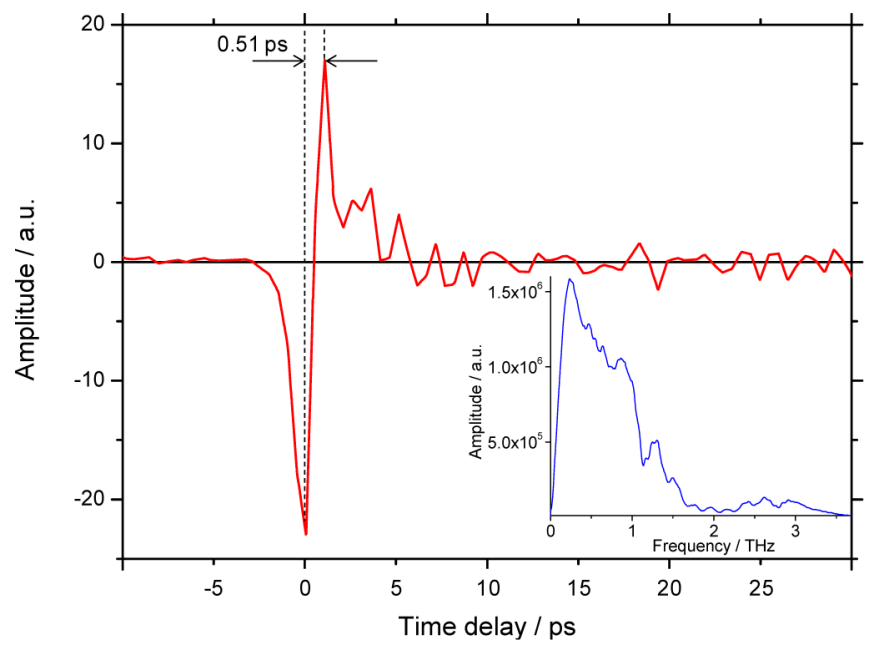

Fig. 3. Temporal waveform of the THz electric field. Inset: corresponding $\mathrm{THz}$ spectrum. 
We then inserted the Hartmann mask at the distance $\mathrm{D}=10 \mathrm{~mm}$ before the crystal in order to perform a complete quantitative evaluation of both the planar and converging wavefronts. First, one has to record a reference image indicating the locations of the mask holes in the plane of the ZnTe crystal, in the absence of the THz beam. This reference image is presented in Fig. 4(a) and can be used for any further THz wavefront measurement. The strong direct reflection from the ZnTe crystal has been removed by slightly tilting this crystal without any visible deviation of the spot positions in the image. Then, Fig. 4(b) (resp. 4(c)) shows the locations of the mask holes in the plane of the crystal in the presence of the planar (resp. converging) $\mathrm{THz}$ beam. Both images have been calculated at $1.2 \mathrm{THz}$ after FFT of the temporal data. However, the inset of Fig. 3, which represents the $\mathrm{THz}$ spectrum transmitted by the ZnTe crystal and Hartmann mask, indicates that our analysis can be extended from 0 to $1.5 \mathrm{THz}$ owing to the large spectral bandwidth of the THz source. Since no significant difference has been observed by changing the frequency of the wavefront analysis from 0 to $1.5 \mathrm{THz}$, we limited our demonstration to $1.2 \mathrm{THz}$ in this paper.
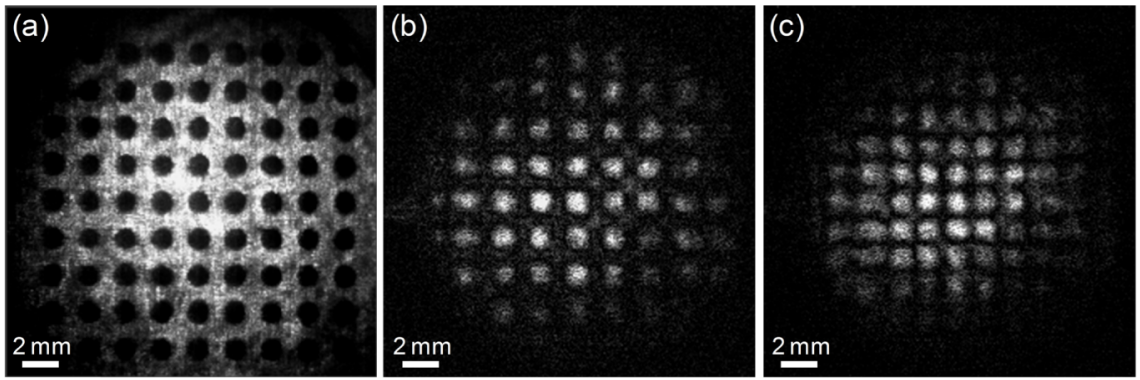

Fig. 4. (a) Reference locations of the mask holes in the plane of the ZnTe crystal. (b) Locations of the projected light spots in the plane of the crystal in the presence of the planar collimated $\mathrm{THz}$ beam. (c) Locations of the projected light spots in the plane of the crystal in the presence of the converging spherical $\mathrm{THz}$ beam.

\section{Wavefront reconstruction and discussion}

The calculation of the wavefront was performed using Igor Pro and ImageJ softwares. For the three images presented in Fig. 4, taking into account the 7 by 7 central spots, the program determines the coordinates of the centroids of each spot which are directly related to the local slopes of the $\mathrm{THz}$ wavefront. It means that the wavefront is reconstructed for a beam diameter of $12 \mathrm{~mm}$. The final calculation of the wavefront is performed by using a least-squares method in which the wavefront $\mathrm{W}(\mathrm{x}, \mathrm{y})$ is represented by a linear combination of the Zernike polynomials [12]:

$$
W(x, y)=\sum_{i} a_{i} Z_{i}(x, y)
$$

where $a_{i}$ represents the amplitudes of the polynomial $Z_{i}$, each polynomial corresponding to one specific aberration. The calculated wavefront of the planar (resp. converging) beam is presented in Fig. 5(a) (resp. 5(b)). The colored scale indicates the local wavefront deformation in $\mu \mathrm{m}$ (accuracy and dynamic range of the measurement will be discussed later). The planar wavefront deformation extends from -50 to $+50 \mu \mathrm{m}(-\lambda / 5$ to $+\lambda / 5$ at $1.2 \mathrm{THz})$ with a visible tilt along the horizontal $\mathrm{x}$-axis. The converging wavefront exhibits a clear spherical shape extending from -500 to $+450 \mu \mathrm{m}(-2 \lambda$ to $+1.8 \lambda$ at $1.2 \mathrm{THz})$, with slightly visible additional aberrations. 

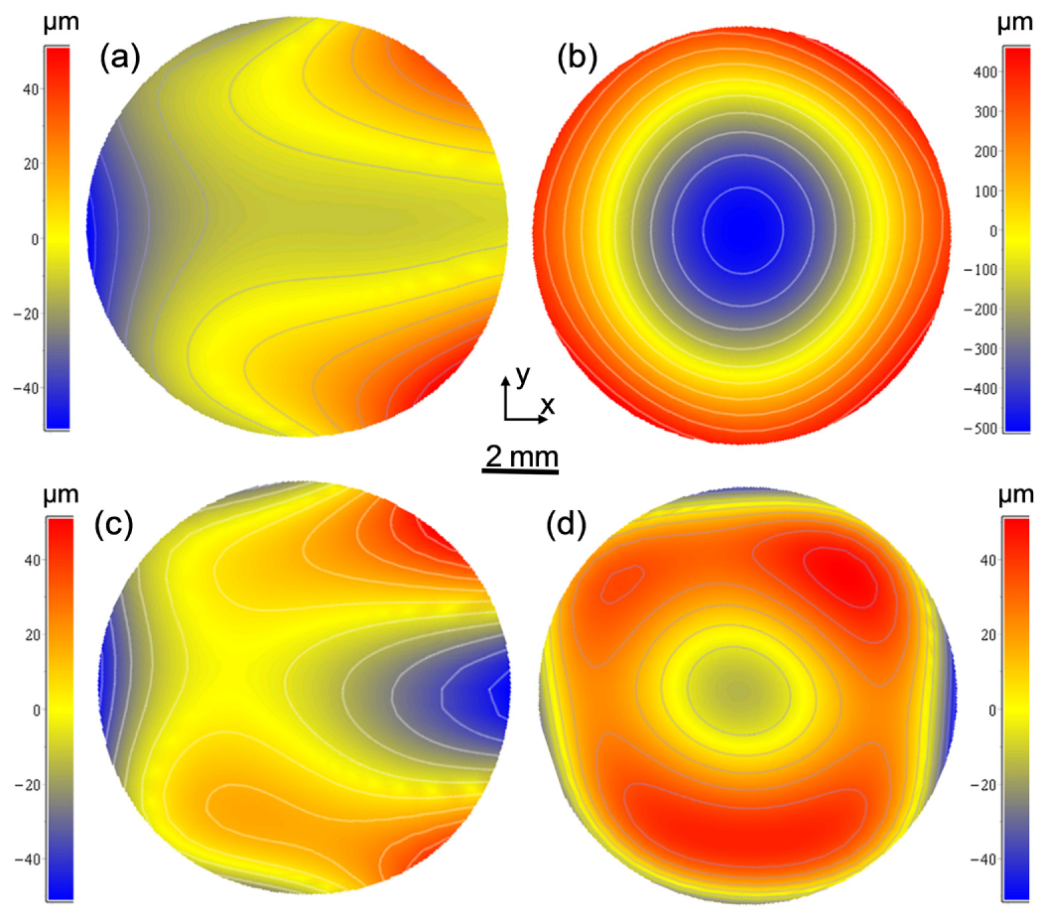

Fig. 5. Reconstructed $\mathrm{THz}$ wavefront. (a) Planar beam. (b) Converging spherical beam. (c) Planar beam without tilt and defocus. (d) Converging spherical beam without tilt and defocus.

A more precise representation of the aberrations can be observed by representing the amplitudes of the 14 first Zernike polynomials (Fig. 6(a) and (b)). The x-tilt amplitude of the planar wavefront is $\mathrm{a}_{2}=18 \mu \mathrm{m}(\lambda / 14$ at $1.2 \mathrm{THz})$. Then, the predominant aberration is the astigmatism along the $\mathrm{x}-$ and $\mathrm{y}$-axis $\left(\mathrm{a}_{5}=-12 \mu \mathrm{m}\right.$ or $-\lambda / 21$ at $\left.1.2 \mathrm{THz}\right)$. For the converging spherical wavefront, it is noteworthy that the same $\mathrm{x}$-tilt is present, which means that the lens is properly aligned since it does not provide any additional wavefront tilt. A large defocus of $\mathrm{a}_{4}=272 \mu \mathrm{m}(1.1 \lambda$ at $1.2 \mathrm{THz})$ is also measured together with the presence of spherical aberration $\left(\mathrm{a}_{12}=-25 \mu \mathrm{m}\right.$ or $-\lambda / 10$ at $\left.1.2 \mathrm{THz}\right)$. Assuming a perfect spherical wavefront (i.e. $\mathrm{a}_{\mathrm{i}}$ $=0$ except for $\mathrm{i}=4)$, the wavefront $\mathrm{W}(\mathrm{r})$ writes:

$$
W(r)=\frac{(\alpha \rho)^{2}}{2 R}=2 \sqrt{3} a_{4} \rho^{2}
$$

where $r=\left(x^{2}+y^{2}\right)^{1 / 2}$ and $\alpha=\mathrm{r} / \rho$ is a normalization factor since the Zernike polynomials are defined for a maximum circular aperture with $\rho=1$. In our measurement, with $\alpha=8.48$ corresponding to the analysis of the 7 by 7 central spots in Fig. 4, one get $R=38 \mathrm{~mm}$, which is close to the theoretical value $\mathrm{f}^{\prime}-\mathrm{e}+\mathrm{D}=45 \mathrm{~mm}$. The $15 \%$ deviation can be attributed to the imperfect spherical wavefront, i.e. the previously noticed presence of $\mathrm{x}$-tilt and spherical aberration.

The first order x-tilt and y-tilt terms are not actually true optical aberrations, as they do not represent a model curvature in the wavefront. Similarly, the second order defocus is directly induced by the presence of the converging lens and is generally not considered for the final analysis of the optical aberrations. We thus plotted again the reconstructed $\mathrm{THz}$ wavefronts of the planar and converging spherical THz beam after fixing to zero the $\mathrm{x}$-tilt, $\mathrm{y}$-tilt and defocus amplitudes (Fig. 5(c) and (d)). At present, the planar wavefront is mainly distorted by the presence of $0^{\circ}$ and $90^{\circ}$ astigmatism $(-12 \mu \mathrm{m})$. The converging spherical wavefront is now 
principally distorted by the primary spherical aberration $(-25 \mu \mathrm{m})$, even if vertical secondary astigmatism $(9 \mu \mathrm{m})$ and vertical quadrafoil $(-9 \mu \mathrm{m})$ are present.

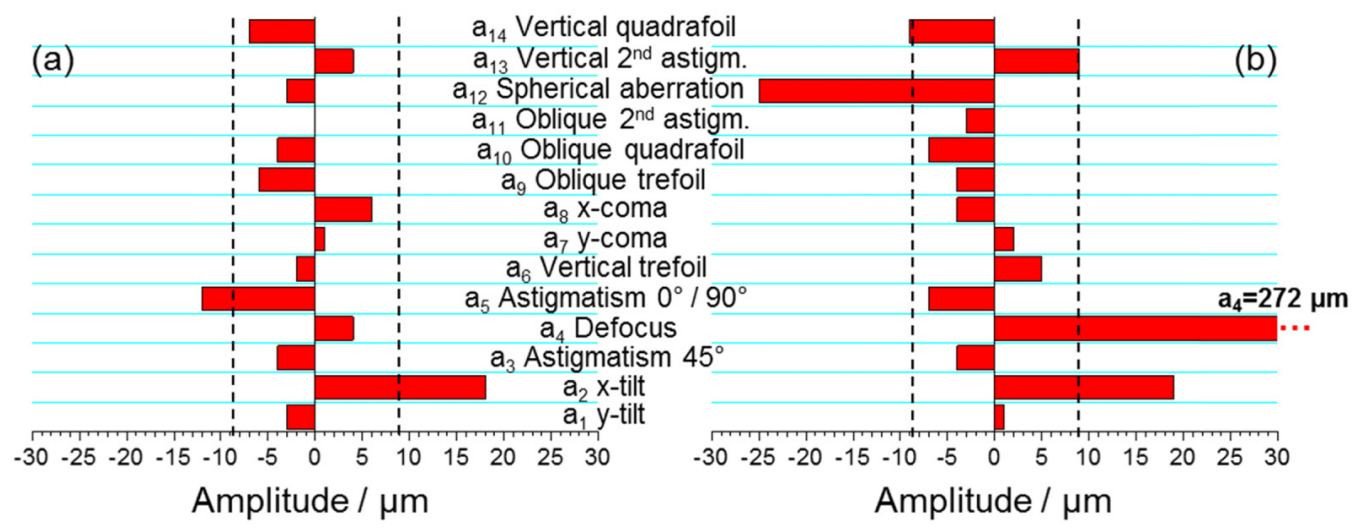

Fig. 6. Amplitudes $a_{i}$ of the Zernike polynomials. (a) Planar $\mathrm{THz}$ wavefront. (b) $\mathrm{THz}$ converging spherical $\mathrm{THz}$ wavefront. The vertical dashed lines show the wavefront measurement accuracy, as calculated in the paper and indicated in Table 1. The amplitude of the defocus term $a_{4}=272 \mu \mathrm{m}$ is out of range of the scale in Fig. $6(\mathrm{~b})$ for a better visualization of the other Zernike terms.

Table 1 summarizes the main characteristics of the $\mathrm{THz}$ wavefront sensor. Let us first recall that it is appropriate to analyze $\mathrm{THz}$ pulses with relatively high peak power since it requires a 2D EO sampling detection with a large aperture ZnTe crystal. The working spectral bandwidth of the sensor extends from 0.1 to $4 \mathrm{THz}$, mainly limited by the $\mathrm{THz}$ absorption of the $\mathrm{ZnTe}$ crystal. In this paper, we demonstrated the wavefront analysis for a single frequency $(1.2 \mathrm{THz})$. However, the analysis can be extended for any other frequency as long as the EO signal can be detected. It is important to note that if the $\mathrm{THz}$ emission spans over a larger spectral range (e.g. THz pulses generated from two color laser-induced air plasma), a 0.1-8 $\mathrm{THz}$ sensitivity could be obtained for instance with a $\mathrm{GaP}$ crystal, with the limitation of the reduced efficiency of the EO effect.

To evaluate the performance of our Hartmann wavefront sensor, we have to consider the characteristics of the mask, the distance $\mathrm{D}=10 \mathrm{~mm}$ between the mask and the ZnTe crystal and the magnification $\gamma=-0.23$ of the imaging lens between the crystal and the camera (Fig. 1(b)). We first calculate the absolute accuracy of the wavefront sensor. It corresponds to the minimum detectable wavefront slope that can be measured by the system. It depends on $\mathrm{D}, \gamma$, the pixel size of the camera, the precision of the centroid determination and the signal-tonoise ratio of the sensor. We consider that the minimum slope corresponds to a spot deviation of 1 pixel $(20 \mu \mathrm{m})$ on the camera sensor. As a consequence, the minimum spot deviation on the $\mathrm{ZnTe}$ crystal is $\Delta x_{\min }=\delta x_{\min } /|\gamma|=87 \mu \mathrm{m}$ and the minimum tilt angle is $\theta_{\min }=\Delta x_{\min } / D=\delta x_{\min } /(|\gamma| D)=0.5^{\circ}$. Considering the $\mathrm{d} / 2=1 \mathrm{~mm}$ diameter of the mask holes, this will provide a local wavefront measurement accuracy $\Delta z_{\min }=2 \theta_{\min } / d=8.7 \mu \mathrm{m}$ $(\lambda / 35$ at $1 \mathrm{THz})$. This parameter is comparable to that of a commercial Hartmann sensor operating in the infrared range. It could be reduced by increasing the magnification factor $\gamma$ or the distance D. This measurement also indicates that a Zernike coefficient with an amplitude smaller than $8.7 \mu \mathrm{m}$ is not significant since it is smaller than the wavefront measurement accuracy $\Delta z_{\min }$. This limit is indicated in Fig. 6 by the vertical dashed lines. It shows that, for the planar wavefront we analyzed in this paper, only $\mathrm{a}_{2}$ (x-tilt) and $\mathrm{a}_{5}\left(0 / 90^{\circ}\right.$ astigmatism) coefficients are really measured, other Zernike coefficients being too small to be detected by our system. For the spherical wavefront, only $\mathrm{a}_{2}$ (x-tilt), $\mathrm{a}_{4}$ (defocus), $\mathrm{a}_{12}$ (spherical aberration), $\mathrm{a}_{13}$ (vertical secondary astigmatism) and $\mathrm{a}_{14}$ (vertical quadrafoil) are significant. 
Table 1. Main characteristics of the THz waveform sensor

\begin{tabular}{ll}
\hline Aperture dimension & $25 \times 25 \mathrm{~mm}^{2}$ \\
Hartmann mask & $9 \times 9$ holes, $1 \mathrm{~mm}$ diameter, \\
& $2 \mathrm{~mm}$ periodicity \\
Working frequency range with ZnTe & $0.1-4 \mathrm{THz}$ \\
detection crystal & \\
Wavefront measurement accuracy & $0.5(\lambda / 25 @ 1 \mathrm{THz})$ \\
Tilt dynamic range & $5.7(3.3 \lambda @ 1 \mathrm{THz})$ \\
\hline
\end{tabular}

We can also evaluate the tilt dynamic range of the sensor which represents the maximum wavefront slope that can be measured. It is generally defined with a spot displacement $\Delta \mathrm{x}_{\max }$ $=\mathrm{d} / 2$ where $\mathrm{d}=2 \mathrm{~mm}$ is the distance between two adjacent holes on the Hartmann mask. The tilt dynamic range is therefore $\theta_{\max }=\Delta x_{\max } / D=5.7^{\circ}$. Considering the $20 \mathrm{~mm}$ clear aperture of the crystal, this will provide a maximum wavefront tilt of $1 \mathrm{~mm}(3.3 \lambda$ at $1 \mathrm{THz})$. This tilt is similar to that obtained with a commercial system operating in the visible even if in this case it corresponds to a dynamic range of nearly $1000 \lambda$. The dynamic range of our sensor can be increased by using a hole mask with either a larger spot separation or a smaller product $|\gamma| D$. However, increasing the dynamic range will also decrease the number of Zernike coefficients available to represent the wavefront. Conversely, increasing the dynamic range by shortening $|\gamma| D$ will also decrease the absolute efficiency (larger minimum wavefront tilt).

\section{Conclusion}

We demonstrated the combination of a Hartmann mask, a ZnTe EO crystal and a CMOS camera for the quantitative wavefront determination of $\mathrm{THz}$ pulses. The proposed method is achromatic and can be applied for all frequencies carried by the THz pulses, limited by the $\mathrm{THz}$ absorption of the detection crystal. Further optimization of the technique includes the use of a Shack-Hartmann mask constituted by a THz matrix of lenses in order to improve the accuracy of the centroid spot determination. Future applications of the sensor will be the precise $\mathrm{THz}$ wavefront analysis of intense $\mathrm{THz}$ sources. It is well known that $\mathrm{THz}$ beams generated from two color laser-induced air plasma or optical rectification of femtosecond laser pulses in $\mathrm{LiNbO}_{3}$ crystal can suffer from severe optical aberrations due to the complex nonlinear optical processes involved into the $\mathrm{THz}$ generation [15]. We believe that our $\mathrm{THz}$ wavefront sensor could provide an effective tool to control the $\mathrm{THz}$ emission from these sources. Another interesting application concerns the association of the Hartmann sensor with deformable mirrors in order to open the route to $\mathrm{THz}$ adaptive optics. This will provide a real advance for time-domain (imaging) spectrometers which require a perfect focalization of the $\mathrm{THz}$ beam or any other $\mathrm{THz}$ devices sensitive to wavefront distortions.

\section{Acknowledgments}

The authors thank Junji Tamatani for the machining of the metallic Hartmann mask. They also thank the financial support from CNRS (PICS 6507). 